\title{
Surgical resection of synchronous liver metastases in gastric cancer patients. A propensity score-matched study
}

\author{
Tomaz Jagric ${ }^{1}$, Matjaz Horvat ${ }^{2}$ \\ ${ }^{1}$ Department of General and Abdominal Surgery, University Medical Centre Maribor, Slovenia \\ ${ }^{2}$ Faculty of Medicine, University of Maribor, Slovenia
}

Radiol Oncol 2021; 55(1): 57-65.

Received 25 June 2020

Accepted 15 October 2020

Correspondence to: Assist. Prof. Tomaž Jagrič, M.D., Ph.D., Department of General and Abdominal Surgery, University Medical Centre Maribor; Ljubljanska ulica 5; 2000 Maribor. E-mail: tomaz.jagric@gmail.com

Disclosure: No potential conflicts of interest were disclosed.

Background. The aim of the study was to determine the value of synchronous liver resection in patients with oligometastatic gastric cancer and the prognostic factors in these patients.

Patients and methods. We compared the results of 21 gastric patients with liver metastases and synchronous liver resection (LMR) to 21 propensity score-matched patients with gastric cancer and liver metastases in whom liver resection was not performed (LMO) and to a propensity score-matched control group of 21 patients without liver metastases and stage III and IV resectable gastric cancer (CG).

Results. The overall 5 -year survival of LMR, LMO and CG were $14.3 \%, 0 \%$, and $19 \%$, respectively $(p=0.002)$. Five-year survival was $47.5 \%$ for well-differentiated tumour compared to $0 \%$ in patients with moderate or poor tumour differentiation $(p=0.006$ ). In addition, patients with RO resection and TNM stage NO-1 had a significantly better survival compared to patients with TNM N stage N2-3 (5-year survival: 60\% for N0-1 vs. $7.7 \%$ for N2-3; $p=0.007$ ).

Conclusions. The results presented in the study support synchronous liver resections in gastric patients and provide additional criteria for patient selection.

Key words: gastric cancer; liver metastases; synchronous resection; propensity score

\section{Introduction}

Liver metastases occur in $3.5-14 \%$ of gastric cancer patients. ${ }^{1-8,12,15-17}$ Surgical resection has been shown to be a viable option in selected cases of gastric cancer patients with liver metastases. Since no randomised controlled trials are yet available regarding the treatment of liver metastases in gastric

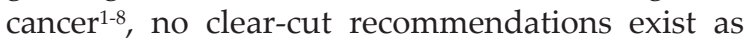
to which patient could benefit the most from such treatment. The aim of our study was to determine the value of synchronous liver resection in patients with oligometastatic gastric cancer and to determine the prognostic factors in these patients. We compared the results of propensity score-matched gastric cancer patients with and without liver re- sections as well as with a control group of stage III and IV patients without liver metastases.

\section{Patients and methods}

\section{Patients}

Patient and tumour characteristics, preoperative diagnostics and laboratory data, types of operations, perioperative complications and mortality from 1546 patient data has been included in our database since 1991. Histopathological descriptions were handled in accordance with the International Union Against Cancer $8^{\text {th }}$ TNM classification of gastric cancer. ${ }^{11}$ Postoperative complications were defined according to the Clavien-Dindo classification. ${ }^{9}$ 
Chemotherapy schemes varied during the study period. The administered preoperative and postoperative regimens included: epirubicin + oxaliplatin, capecitabine + fluorouracil, capecitabine + oxaliplatin, or 5-fluorouracil + leucovorin. For our analysis, only the patients who had synchronous liver metastases and gastric resection with curative intent (LMR) were selected. The results of these operations were compared to propensity score-matched (PSM) patients with liver metastases who had gastric cancer resection, in whom the surgeon did not opt for additional liver resection (LM0). The decision regarding liver metastasis resection was obtained on the preoperative tumour board and during the operation at surgeon's discretion. Patients were selected for liver resection, if they met the following criteria: (i) three or less metastases (oligometastatic gastric cancer); (ii) no distant metastases; (iii) resectable primary tumour; (iv) good general health. The nomenclature of hepatic anatomy and resection was used according to the Brisbane 2000 system. ${ }^{10}$ Since we could not determine retrospectively whether patients' general state or advanced disease precluded liver metastases resection in LM0 group, we selected an additional PSM control group (CG). For CG, the propensity score-matched stage III and IV gastric cancer patients without having liver metastases resected with curative intent were used for estimation of treatment benefit. Follow-up was carried out by surgeons and oncologists. Informed consent was obtained from all individual participants included in the study. The study was approved by the local ethics committee of the University Medical Centre Maribor in Slovenia (UKC-MB-KME-58/20).

\section{Propensity score-matching}

The data from 1546 patients prospectively stored in our database was used for PSM. Patients were matched using the propensity score method as described by Rosenbaum and Rubin. ${ }^{18,19}$ The propensity score for an individual was calculated on the given covariates of preoperative haemoglobin levels, age, American Society for Anaesthesiology (ASA) score and the International Union against Cancer (UICC) stage using the multivariate logistic regression model. With this method three groups of PSM patients containing 21 patients each was formed: (i) Patients with liver metastases and no liver resection (LM0); (ii) Patients with liver metastases and liver resection (LMR); (iii) Patients with stage III and IV gastric cancer and no liver resection (CG). In all patients a gastrectomy with local curative intent was performed.

\section{Statistical analysis}

Continuous variables were expressed as mean \pm standard deviation (SD) and categorical variables as percentage. Continuous variables were compared with Student's t-test for normally distributed variables; nonparametric variables were tested with Mann-Whitney's U-test. The cut-off levels of continuous variables were determined by means of receiver operator curves with cut-off value of AUC above 0.75 and $p$ value of less than 0.05 . Variables above the threshold $p$ value of 0.1 were included for multivariate analysis. The Cox regression model was used for primary analysis. Estimates of treatment effect were expressed as hazard ratios with $95 \%$ confidence interval. Kaplan-Meier curves were constructed to determine time-to-event endpoints. Differences in survivals between groups were determined with the Log-rank and Breslow tests. $\mathrm{P}$ value of $>0.05$ was selected as the level of significance. All statistical analyses were performed on SPSS for Windows 10 v. 22 (IBM).

\section{Results}

\section{Patients}

In the final analysis 63 patients resected for gastric cancer were included. The characteristics of these patients, their tumours, and operations are provided in Table 1. All three groups were balanced according to age, gender, tumour location, operations, UICC and TNM stage and number of resected lymph nodes. In the LM0 group, significantly less patients received chemotherapy compared to LMR and CG group ( $4.8 \%$ vs. $42.9 \%$ vs. $42.9 \%$; p $=0.004)$. A D2 lymphadenectomy was performed significantly more often in the LMR and CG compared to patients in the LM0 group (LM0: 19\%, LMR: $76.2 \%$ and CG: $81 \% ; p<0.0001)$. The number of harvested lymph nodes (LNs) per operation was significantly higher in LMR and CG compared to LM0 group (LM0: $18 \pm 9$ LNs, LMR: $27.6 \pm 14.6$ LNs; CG: $26 \pm 17 \mathrm{LNs} ; p=0.028)$. All patients in LM0 had locally microscopically negative resection margins. In LMR patients a R0 resection could be obtained in $85.7 \%$ compared to $100 \%$ in the CG $(p=0.076)$. LM0 patients had significantly more liver metastases compared to LMR patients $(p<0.0001)$. Most of LM0 patients had more than three metastases $(71.4 \%)$ compared to one liver metastasis usually present in LMR patients (90.5\%). Patients with liver metastases (LM0 and LMR) had a tumour location almost exclusively in the middle and lower third 
TABLE 1. Patients', tumour and operations characteristics of the included patients

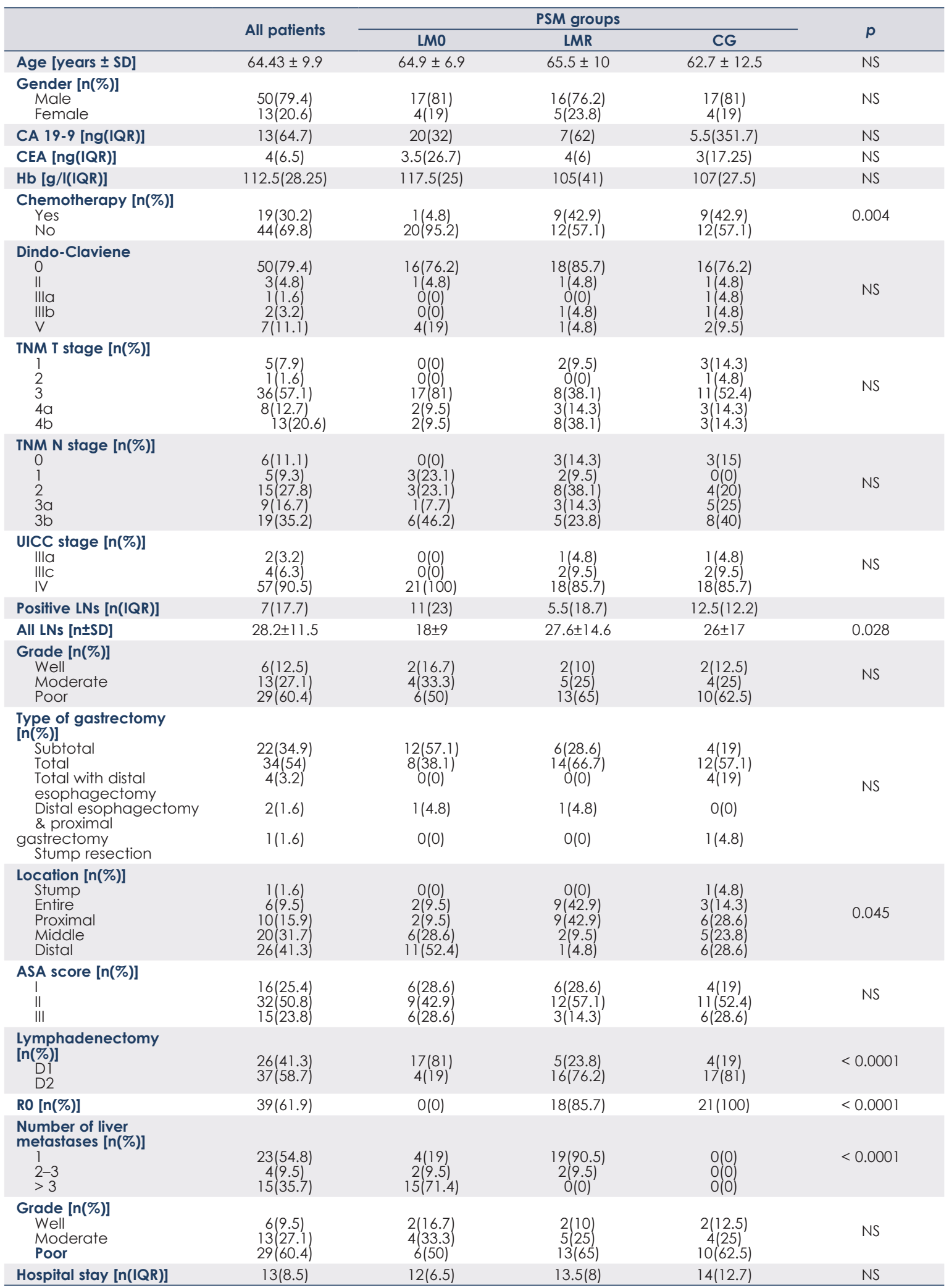

$\mathrm{ASA}=$ American Society for Anaesthesiology; Ca 19-9 = carbohydrate antigen 19-9; CEA = carcinoembryonic antigen; CG = patients without liver metastases and stage III and IV resectable gastric cancer; $\mathrm{Hb}=$ serum hemoglobin levels; IQR = interquartile range; LMO = patients with gastric cancer and liver metastases in whom liver resection was not performed; LMR = patients with synchronous liver metastases and gastric resection with curative intent; LNS = lymph nodes; NS = not significant; PSM = propensity score-matched; UICC = International Union against Cancer 


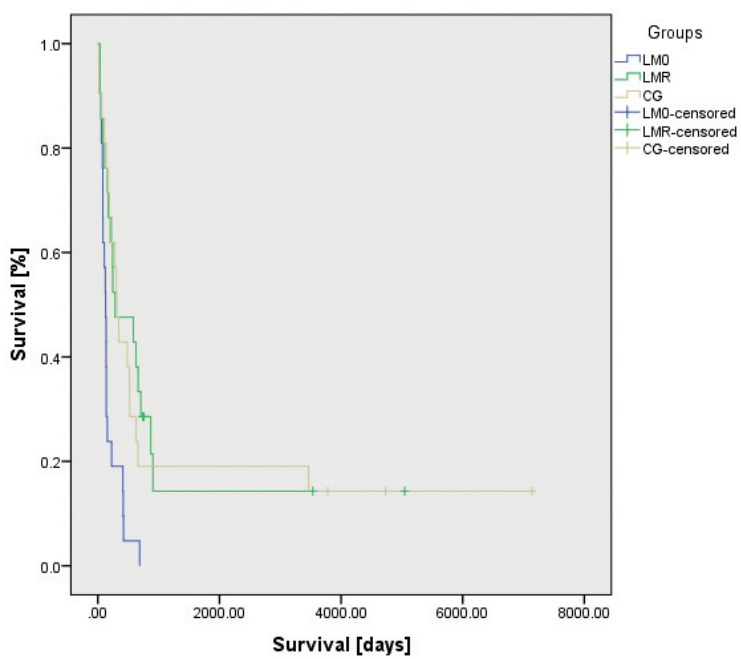

FIGURE 1. Cumulative survivals of patients with liver metastases without resection, with liver resection and in the control group respectively.

TABLE 2. Characteristics of liver resections in the group of patients with synchronous liver metastases and gastric resection with curative intent (LMR)

\begin{tabular}{lc}
\hline & $n(\%)$ \\
\hline Segment involvement & \\
II & $0(0)$ \\
II/III & $2(8.6)$ \\
III & $3(13)$ \\
IV & $9(39.1)$ \\
V & $2(8.6)$ \\
VI & $2(8.6)$ \\
VII & $0(0)$ \\
VIII & $3(13)$ \\
Liver resection & $2(8.6)$ \\
Metastasectomia of 1 metastasis & \\
Metastasectomia of >1 metastasis & $12(57.1)$ \\
Segmental resection & $3(14.3)$ \\
Hepatectomy & $3(14.3)$ \\
Radio frequency ablation & $1(4.8)$ \\
Dindo-Claviene morbidity & $2(9.5)$ \\
OI & \\
II & $18(85.7)$ \\
IIIa & $1(4.8)$ \\
IIIb & $0(0)$ \\
V & $1(4.8)$ \\
Number of metastases & $1(4.8)$ \\
I metastasis & \\
2-3 metastasis & $19(90.5)$ \\
$>$ 3 metastasis & $2(9.5)$ \\
\hline
\end{tabular}

of the stomach, while the tumour location in CG was evenly distributed in the whole stomach $(\mathrm{p}=$ 0.045).

\section{Surgery, morbidity and mortality}

There were no significant differences in the perioperative mortality between the three groups. The perioperative morbidity in the LM0, LMR and CG was $23.8 \%, 14.3 \%$, and $23.8 \%$, respectively. Major morbidity (Dindo-Claviene $>\mathrm{IIIb}$ ) was observed in $16.7 \%, 9.6 \%$, and $14.3 \%$ in the LM0, LMR and CG group, respectively. The 30-day mortality was $9.5 \%, 4.8 \%$ and $9.5 \%$ in the LM0, LMR, and CG group, respectively. Although the differences were insignificant, the patients in the LMR group had the lowest perioperative morbidity and mortality. The results of surgical treatment are presented in Table 1.

The characteristics of liver resections in LMR group are presented in Table 2. Metastasectomy of single metastasis was performed in most cases $(57.1 \%)$, followed by segmental resection of a single metastasis $(14.3 \%)$ and metastasectomy of more than 1 metastasis (14.3\%). Major resection was performed only in one case $(4.8 \%)$, and radio frequency ablation was performed in two cases $(9.5 \%)$. The liver resections for 21 included cases, the involved liver segments and recurrence sites are documented in Table 3. The most frequent recurrence site was the liver followed by peritoneum. Notably $47.6 \%$ of cases did not have a documented recurrence after liver resection.

In Table 4 the types of operations in the CG are presented. Additional resection of an involved organ was undertaken in $47.6 \%$ of cases. The most commonly infiltrated other organ was the tail of the pancreas ( 2 cases) and the local peritoneum of the bursa omentalis (8 cases). The most frequent additional resection in CG was the local peritonectomy, followed by left splenopancreatectomy with segmental colon resection and left splenopancreatectomy with left adrenal resection. Left pancreatectomy was associated with most morbidity, while mortality was the highest in patients with no additional resection.

\section{Multivariate analysis}

From the included predictors, age, gender, ASA score, Ca 19-9 serum levels, haemoglobin serum levels, additional liver resection, tumour grade, UICC stage, and TNM nodal stage were significantly associated with survival. The hazard ratios, 95\% confidence intervals, and the p-values are listed in Table 5.

\section{Survival analysis}

None of the patients in the LM0 group survived 5 years. Their survival was significantly shorter compared to patients in the LMR group a cumulative 

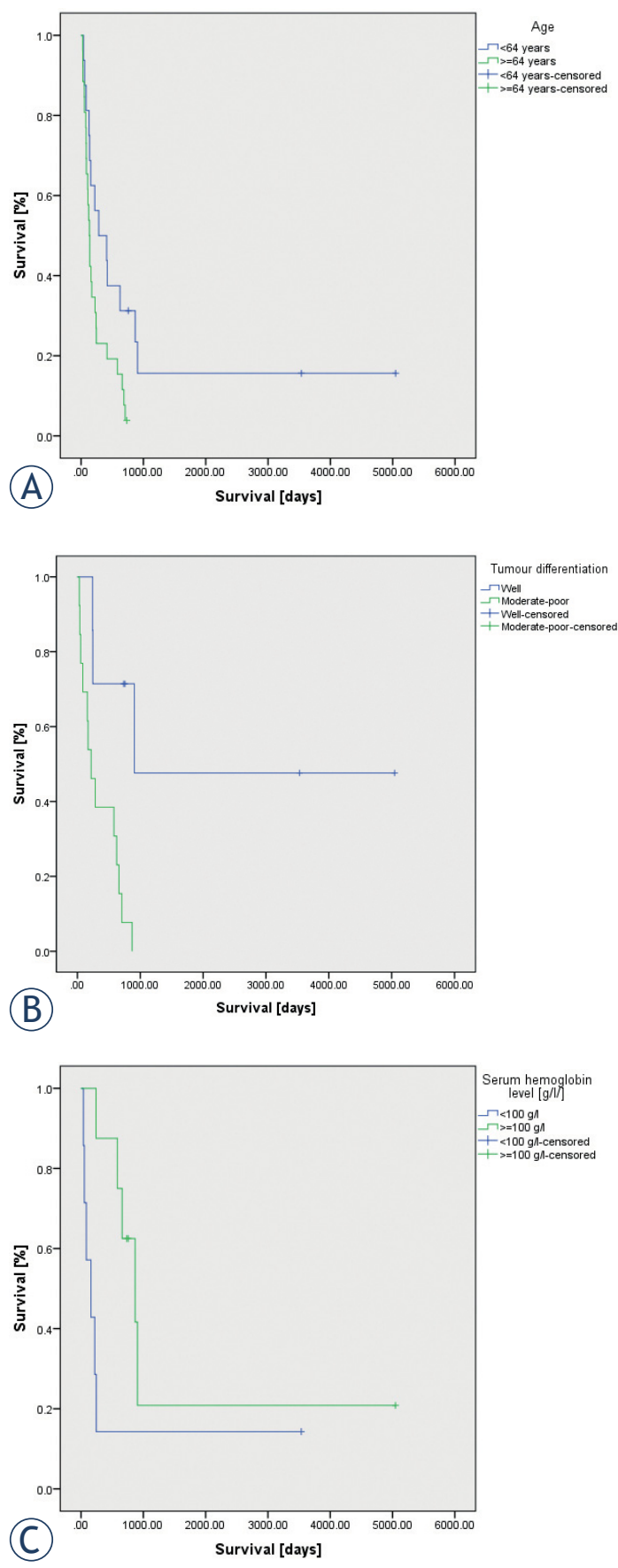

5-year survival of $14.3 \%(\mathrm{p}=0.002)$. The survival of LMR patients was comparable to the survival of CG patients who had a 5 -year survival rate of $19 \%$. The median survival was 4.2 months, 9.3 months and 10.2 moths in the LM0, LMR and CG group respectively. The survival plots for the cumulative survival of patients in each group are shown in Figure 1 .
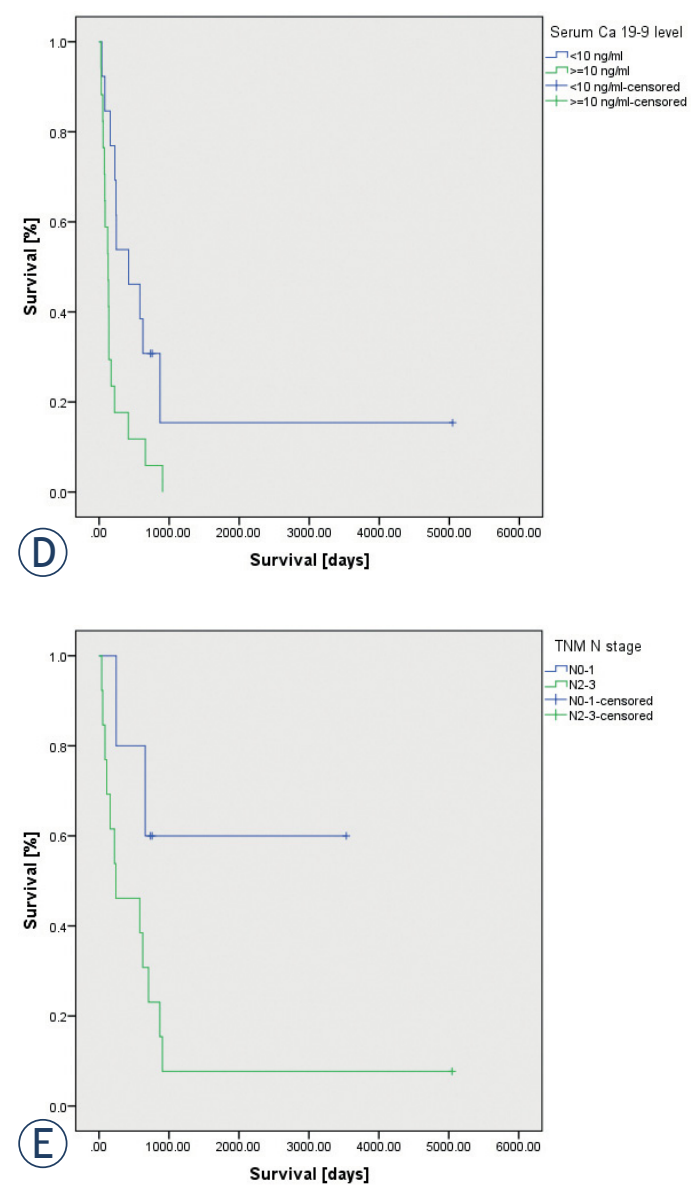

FIGURE 2. Survival of subgroups. Overall survival according age (A), tumour differentiation (B), serum CA-19 level (C), N stage of TNM (D) and serum haemoglobin (E).

The multivariate analysis identified age, gender, ASA score, carbohydrate antigen 19-9 (Ca 19-9) serum levels, haemoglobin serum levels, additional liver resection, tumour grade, UICC stage, and TNM nodal stage as significant predictors for survival. These predictors were used to determine cutoff levels and determine the subgroup of patients with the greatest benefit of liver resection. With the ROC analysis we determined the cut-off for serum levels of Ca19-9, age, haemoglobin serum levels. Patients with Ca19-9 levels above $10 \mathrm{ng} / \mathrm{ml}$ had significantly worse survival (5-year survival: $0 \%$ vs. $15.4 \% ; p=0.003)$. The survival advantage was lost for R0 resections. Patients with TNM stage N0-1 had an insignificantly better survival compared to patients with TNM stage N2-3. If only R0 patients were included, patients with TNM stage N0-1 had a significantly better survival compared to patients with TNM N stage N2-3 (5-year survival: $60 \%$ for N0-1 vs. $7.7 \%$ for N2-3; $p=0.007)$. Patients with a 
TABLE 3. Characteristics of liver metastases and recurrence sites in the group of patients with synchronous liver metastases and gastric resection with curative intent (LMR)

\begin{tabular}{|c|c|c|c|c|}
\hline Case No. & $\begin{array}{l}\text { Involved } \\
\text { segment }\end{array}$ & $\begin{array}{l}\text { Number of } \\
\text { metastases }\end{array}$ & Type of resection & Recurrence site \\
\hline 1 & $\|$ & 1 & Segmentectomy & 0 \\
\hline 2 & IV & 1 & Metastasectomy & 0 \\
\hline 3 & III & 1 & Segmentectomy & $\begin{array}{c}\text { Peritoneal } \\
\text { carcinomatosis }\end{array}$ \\
\hline 4 & $\|/ I\|$ & 1 & Segmentectomy & Liver metastases \\
\hline 5 & III & 1 & Metastasectomy & 0 \\
\hline 6 & VII & 1 & Metastasectomy & 0 \\
\hline 7 & III & 1 & Metastasectomy & $\begin{array}{l}\text { Local } \\
\text { recurrence }\end{array}$ \\
\hline 8 & III & 1 & Segmentectomy & $\begin{array}{c}\text { Peritoneal } \\
\text { carcinomatosis }\end{array}$ \\
\hline 9 & VIII & 1 & Metastasectomy & Liver metastases \\
\hline 10 & III & 1 & Segmentectomy & 0 \\
\hline 11 & V & 1 & Metastasectomy & $\begin{array}{l}\text { Local } \\
\text { recurrence }\end{array}$ \\
\hline 12 & IV, II & 3 & Metastasectomy & Liver metastases \\
\hline 13 & $\|/\| \|$ & 1 & Metastasectomy & Liver metastases \\
\hline 14 & III & 1 & Metastasectomy & 0 \\
\hline 15 & $\|/\| \|$ & 1 & $\begin{array}{c}\text { Left lateral } \\
\text { secienectomy }\end{array}$ & 0 \\
\hline 16 & III & 1 & Metastasectomy & 0 \\
\hline 17 & III & 1 & Metastasectomy & $\begin{array}{c}\text { Peritoneal } \\
\text { carcinomatosis }\end{array}$ \\
\hline 18 & V, VIII & 2 & Metastasectomy & Liver metastases \\
\hline 19 & VII & 1 & $\begin{array}{l}\text { Radio frequency } \\
\text { ablation }\end{array}$ & $\begin{array}{l}\text { Liver metastases } \\
\text { and peritoneal } \\
\text { carcinomatosis }\end{array}$ \\
\hline 20 & VII & 1 & $\begin{array}{l}\text { Radio frequency } \\
\text { ablation }\end{array}$ & 0 \\
\hline 21 & III & 1 & Segmentectomy & 0 \\
\hline
\end{tabular}

TABLE 4. Morbidity and mortality of additional resections in in the control group of patients without liver metastases and stage III and IV resectable gastric cancer (CG)

\begin{tabular}{lccc}
\hline & N (\%) & Morbidity (\%) & Mortality (\%) \\
\hline $\begin{array}{l}\text { Gastrectomy } \\
\text { Subtotal }\end{array}$ & $4(19)$ & $0(0)$ & $1(25)$ \\
$\quad$ Total & $12(57.1)$ & $2(16.6)$ & $1(25)$ \\
$\quad$ Total with distal esophagectomy & $4(19)$ & $1(25)$ & $0(0)$ \\
$\quad$ Stump resection & $1(4.8)$ & $0(0)$ & $0(0)$ \\
$\begin{array}{l}\text { Additional resection } \\
\text { No additional resection }\end{array}$ & $11(52.4)$ & $1(9.1)$ & $2(18.2)$ \\
$\begin{array}{l}\text { Local peritonectomy } \\
\text { Left splenopancreatectomy and }\end{array}$ & $8(4.8)$ & $1(12.5)$ & $0(0)$ \\
segmental colon resection & $1(4.8)$ & $0(0)$ & $0(0)$ \\
$\quad$ Left splenopancreatectomy and & $1(4.8)$ & $1(100)$ & $0(0)$ \\
left adrenal resection & & &
\end{tabular}

well differentiated tumour had a significantly better survival compared to patients with moderate and poor tumour differentiation. This difference was more pronounced in patients with R0 resection. Five-year survival in these patients was $47.5 \%$ for well-differentiated tumours compared to $0 \%$ in patients with moderate or poor tumour differentiation $(p=0.006)$. Patients younger than 64 years had a 5-year survival of $15.6 \%$ compared to $3.8 \%$ in patients older than 64 years $(p=0.029)$. R0 resected patients with serum haemoglobin levels below $100 \mathrm{~g} / \mathrm{l}$ before the operation had a significantly worse 5-year survival compared to patients with higher haemoglobin levels (5-year survival: 14.3\% vs. $20.8 \% ; p=0.09$ ). The multivariate analysis also determined gender and ASA score to be significant predictors for survival; however, we could not determine any cut-off levels for patient stratification. The survival plots of different subgroups are presented in Figure 2.

\section{Discussion}

Liver metastases in gastric cancer patients usually occur as part of a systemic failure and rarely present as isolated disease suitable for resection. ${ }^{1-8,12,15-17}$ In the present study liver resection has only been performed in $1.36 \%$ of patients operated for gastric cancer. As a result of low incidence of resectable liver metastases prospective large-scale randomised controlled trials are not feasible and at present no clear-cut recommendations exist for liver resection of gastric cancer metastases. ${ }^{1-8,12,15-17}$ To evaluate potential benefits and prognostic factors for synchronous liver metastases resections in gastric cancer patients we performed a retrospective propensity score-matched study.

The treatment of stage IV gastric cancer patients is still the subject of heated debates. The results of the REGATTA trial suggest that palliative resection in addition to chemotherapy does not improve survival for stage IV gastric cancer patients compared to chemotherapy alone, putting in question the value of surgery in oligometastatic gastric cancer. ${ }^{21}$ However, in the REGATTA trail only palliative gastrectomies were performed limiting the value of these results in gastric cancer patients with potentially resectable liver metastases. In the light of emerging evidence proving benefits of liver mestastases resections in oligometastatic gastric cancer patients, we performed a retrospective analysis of stage IV gastric cancer patients with liver metastases. The data from the present study is in accordance with the results of studies that show a significant increase in long-term survival in patients with liver resections in gastric cancer patients. . $^{1-8,12,15-17}$ 
The multivariate analysis confirmed that liver resection is a significant predictor for long-term survival. Median overall survival of 4.2 months in LM0 group was similar to other studies that reported median survival of 0 to 15 months with unresectable liver metastases. ${ }^{8}$ In contrast, the median overall survival in LMR group was significantly longer compared to LM0 group. In our study longterm survival of gastric cancer patients was only possible when liver metastases were resected.

The comparison of results from the LM0 and LMR group might support the claim that liver resections have a positive impact on long-term survival $^{8,12,15-17}$; however, the retrospective nature of the study carries some risk of biased selection despite the PSM. At least six patients in LM0 group had potentially resectable metastases. Because we could not determine retrospectively the precise reasons for unresectability of liver metastases in these LM0 patients, local advanced stage, poor general state, anatomical location of the metastasis or some other factor not included in the propensity score calculation might have precluded a safe resection the time of operation. In order to better evaluate the potential benefit of liver resection in gastric cancer patients we compared the results of LMR patients to PSM control group of UICC stage III and IV gastric cancer patients (CG). In both groups, patients were well-balanced according to patients' and tumour characteristics. In addition, no difference was observed in the adjuvant treatment between LMR and CG group. The long-term survival of patients in LMR was comparable to patients in CG. Based on these results, we concluded that liver resection offered a survival benefit comparable to propensity score-matched patients with stage III and IV disease a $\mathrm{R} 0$ resection and without liver metastases.

An important aspect of treatment effect evaluation is the safety of the procedure. The perioperative morbidity and mortality in the LMR group was $14.3 \%$ and $4.8 \%$ that compares favourably to results published in other studies. ${ }^{1-8,12-17}$ Notably, perioperative morbidity in LMR group was significantly better compared to CG group. This discrepancy in perioperative morbidity could in part be explained in the additional multivisceral resections in the CG. Pancreatic resections have been identified in previous studies to increase morbidity and mortality. ${ }^{13,14}$ In fact, we observed that the pancreas tail resection was associated with $50 \%$ morbidity in the CG. This might explain a lower complication rate in the LMR group compared to CG group.

The 5-year survival of LMR patients in our study was $14.3 \%$ which was comparable to other studies
TABLE 5. Cox proportional hazard model for survival after gastric cancer resection with liver metastases

\begin{tabular}{lcccc} 
& \multirow{2}{*}{ HR } & \multicolumn{2}{c}{$95 \% \mathrm{Cl}$} & \multirow{2}{*}{$\mathbf{p}$} \\
\cline { 3 - 4 } & & Lower & Upper & \\
\hline Age & 1.497 & 1.213 & 1.846 & $<0.0001$ \\
\hline Gender & 55.237 & 4.626 & 659.594 & 0.002 \\
\hline ASA score & 0.049 & 0.009 & 0.261 & $<0.0001$ \\
\hline Ca 19-9 & 1 & 1 & 1.001 & 0.02 \\
\hline Hb & 0.918 & 0.864 & 0.974 & 0.005 \\
\hline Additional liver resection & 0.001 & 0 & 0.029 & $<0.0001$ \\
\cline { 1 - 4 } Tumour grade & 8.276 & 1.971 & 34.753 & 0.004 \\
\hline UICC stage & 0 & 0 & 0.003 & 0.002 \\
\hline TNM N stage & 0.386 & 0.163 & 0.917 & 0.031 \\
\hline
\end{tabular}

ASA = American Society for Anaesthesiology; $\mathrm{Ca} 19-9=$ carbohydrate antigen 19-9; $\mathrm{Cl}=$ confidence interval; $\mathrm{Hb}=$ serum hemoglobin levels [ $\mathrm{g} / \mathrm{l}] ; \mathrm{HR}=$ hazard ratio; $\mathrm{UICC}=$ International Union against Cancer

that reported the 5 -year survival of $0 \%$ to $42 \%$ after liver resection..$^{1-8,12,15-17}$ In their seminal multi-centric retrospective analysis of stage IV gastric cancer in Western patients Ministrini et al. reported 5-year survival of $11.8 \%{ }^{22}$ Although our results compare favourably to results of Ministrini et al., some studies reported better long-term survival of up to $32 \%$ to $59 \% .{ }^{12,20}$ An important reason for better survival after liver resection in these repots might be that patients with metachronous liver resections were included. Metachronous resections have been determined to be a significant prognostic factor. ${ }^{7}$ In other studies patients proceeded to liver resection only after a course of chemotherapy, while patients in whom disease progression was determined might have been excluded. In the present report, only synchronous resections have been analysed. It was therefore impossible to exclude patients at risk for progression. In fact, patient selection is the most critical aspect of liver metastases treatment in gastric cancer patients. Unfortunately, no clear recommendations for patients' selection exist yet. In our study, the multivariate analysis identified age, gender, ASA score, Ca 19-9 serum levels, haemoglobin serum levels, additional liver resection, tumour grade, UICC stage, and TNM nodal stage as significant predictors for long-term survival in LMR patients. The most powerful predictor for long-term survival was the TNM N stage of the primary tumour. The 5-year survival of LMR patients with N0-1 stage was $47.5 \%$ and even $60 \%$ in patients with TNM stage N0-1 and R0 resection. These results are comparable even to results 
published by Tasubayashi et al. who reported a 5 -year overall survival of $59 \% .^{12}$ Our results suggest that patients with a higher nodal stage of the primary tumour were at higher risk for systemic recurrence. They would probably progress after adjuvant treatment and would not be candidates for metachronous resection. Therefore, high nodal stage might be a valuable negative selection criterion when considering synchronous liver resection.

As we further stratified patients, we determined additional subgroups with the greatest survival benefit. Patients with serum levels of Ca 199 below $10 \mathrm{ng} / \mathrm{ml}$ had a 5-year survival of $15.4 \%$. Similar long-term survival was observed in patients younger than 64 years $(15.6 \%)$. Patients with preoperative haemoglobin levels of more than 100 $\mathrm{g} / 1$ had a $5 Y S$ of $20.8 \%$. Next to the TNM N stage, tumour grade was found to have the most significant impact on long-term survival. Patients with a well-differentiated tumour had a 5-year survival of $47.5 \%$ compared to $0 \%$ in moderate to poor differentiated tumour. As these predictors are linked to inherent tumour biology, probably the most important determinants for behaviour of liver metastases are intrinsic tumour properties that have yet to be determined in future studies.

Our study has some limitations. It is a retrospective study with a limited number of included patients. Secondly, although the patients were selected with propensity score-matching, some selection bias could have still been present, since not all selection criteria have been included in the propensity score calculation. And finally, only synchronous metastases have been included in the analysis. Conversely, since isolated liver metastases occur only in small number of resectable gastric cancer, most of the presently published studies have only a small number of cases. Still, these studies have a significant merit as they help build recommendations for the treatment of liver metastases in gastric cancer patients. In the present paper we could show that synchronous liver resection in selected cases is beneficial since patients might expect similar long-term survival as R0 resected stage IV gastric cancer patients without liver metastases. The liver resection was identified as an independent prognostic factor on multivariate analysis. Based on results of our study, we determined selection criteria for liver resection of synchronous gastric cancer metastases.

\section{Conclusions}

In conclusion, our results confirm that synchronous liver resection in gastric cancer patients is safe and offers significant survival benefit compared to chemotherapy alone. Moreover, after resection of liver metastases with curative intent, patients might expect similar long-term survival as PSM patients with stage III and IV gastric cancer without liver metastases and $\mathrm{R} 0$ resection. In addition, we determined that patients benefiting the most from synchronous liver resection are patients younger than 64 years, with less than three liver metastases, Ca 19-9 serum levels below $10 \mathrm{ng} / \mathrm{ml}$, well-differentiated primary tumours, and TNM N0-1 stage, provided an $\mathrm{R} 0$ resection can be obtained. Future prospective randomized studies will have to confirm the value of these selection factors to provide clear-cut guidelines for treatment of gastric cancer patients with liver metastases.

\section{References}

1. Takemura N, Saiura A, Koga R, Arita J, Yoshioka R, Ono Y, et al. Long-term outcomes after surgical resection for gastric cancer liver metastasis: an analysis of 64 macroscopically complete resections. Langenbecks Arch Surg 2012; 397: 951-7. doi: 10.10007/s00423-012-0959-z

2. Sarel Al, Yelluri S. Gastric adenocarcinoma with distant metastasis: is gastrectomy necessary? Arch Surg 2007; 142: 14349. doi: 10.1001/archsurg.142.2.143

3. Bang YJ, Van Cutsem E, Feyereislova A, Chung HC, Shen L, Sawaki A, et al. Transtuzumab in combination with chemotherapy versus chemotherapy alone for treatment of HER2-positive advanced gastric or gastro-esophageal junction cancer (ToGa): a phase 3, open-label, randomised controlled trial. Lancet 2010; 37: 687-97. doi: 10.1016/s0140-6736(10)61121-x

4. Kataoka K, Kinoshita T, Moehler M, Mauer M, Shitara K, Wagner AD, et al, On behalf of EORTIC GITCG Group and JCOG SCGS Group. Current management of liver metastases from gastric cancer: what is common practise? New challenge of EORTIC and JCOG. Gastric Cancer 2017; 20: 904-12. doi: 10.1007/s10120-017-0696-7

5. Kinoshita T, Kinoshita T, Saiua A, Esaki M, Sakamoto H, Yamanaka T. Multicentre analysis of long-term outcome after surgical resection for gastric cancer liver metastases. Br J Surg 2015; 102: 102-7. doi: 10.1002/ bjs.9684

6. Zacherl J, Zacherl M, Scheuba C, Steininger R, Wenzel E, Mühlbacher Jakesz $\mathrm{R}$, et al. Analysis of hepatic resection of metastasis originating from gastric adenocarcinoma. J Gastrointestinal Surg 2002; 6: 682-9. doi: 10.1016/ s1091-255x(01)00075-0

7. Schildberg CW, Croner R, Merkel S, Schellerer V, Müller V, Yedibela S, et al. Outcome of operative therapy of hepatic metastatic stomach carcinoma: A retrospective analysis. World J Surg 2012; 36: 872-8. doi: 10.1007/s00268012-1492-5

8. Sakamoto $\mathrm{Y}$, Sano T, Shimada K, Esaki M, Saka M, Fukagawa T, et al. Favorable indications for hepatectomy in patients with liver metastasis from gastric cancer. J Surg Oncol 2007; 95: 534-9. doi: 10.1002/jso.20739

9. Clavien PA, Barkun J, de Oliveira ML, Vauthey JN, Dindo D, Schulick RD, et al. The Clavien-Dindo classification of surgical complications: five-year experience. Ann Surg 2009; 250: 187-96. doi: 10.1097/SLA.0b013e3181b13ca2 
10. Strasberg SM. Nomenclature of hepatic anatomy and resections: a review of the Brisbane 2000 system. J Hepatobiliary Surg 2005; 12: 351-5. doi: $10.1007 / \mathrm{s} 00534-005-0999-7$

11. Jiu-Yang Liu, Chun-Wei Peng, Xiao-Jun Yang, Chao-Qun Huang, Yan Li. The prognosis role of AJCC/UICC 8th Edition staging system in gastric cancer, a retrospective analysis. Am J Transl Res 2018; 10: 292-303. PMID: 29423014

12. Tatsubayashi T, Tanizawa Y, Miki Y, Tokunaga M, Bando E, Kawamura T, et al. Treatment outcomes of hepatectomy for liver metastases of gastric cancer diagnosed using contrast-enhanced magnetic resonance imaging. Gastric Cancer 2017; 20: 387-93. doi: 10.1007/s10120-016-0611-7

13. Hartgrink $\mathrm{HH}$, van de Velde $\mathrm{CJ}$, Putter $\mathrm{H}$, Bonenkamp JJ, Klein Kranenbarg E, Songun I, et al. Extended lymph node dissection for gastric cancer: who may benefit? Final results of the randomized Dutch gastric cancer group trial. J Clin Oncol 2004; 22: 2069-77. doi: 10.1200/JCO.2004.08.026

14. Cuschieri A, Fayers P, Fielding J, Craven J, Bancewicz J, Joypaul V, et al. Postoperative morbidity and mortality after D1 and D2 resections for gastric cancer: Preliminary results of the MRC randomised controlled surgical trial. The surgical cooperative group. Lancet 1996; 347: 995-9. doi: 10.1016/ s0140-6736(96)90144-0

15. Jung JO, Nienhüser $\mathrm{H}$, Schleussner $\mathrm{N}$, Schmidt T. Oligometastatic gastroesophageal adenocarcinoma: molecular pathophysiology and current therapeutic approach. Inter J Mol Sciences 2020; 21: 3-24. doi: 10.3390/ ijms21030951

16. Oki E, Tokunaga S, Emi $Y$, Kusumoto $T$, Yamamoto $M$, Fukuzawa $K$, et al. Kyushu Study Group of Clinical Cancer. Surgical treatment of liver metastasis of gastric cancer: a retrospective multicenter cohort study (KSCC1302). Gastric Cancer 2016; 19: 968-76. doi: 10.1007/s10120-015-0530-z

17. Fujitani K, Yang HK, Mizusawa J, Kim YW, Terashima M, Han SU, et al. Gastrectomy plus chemotherapy versus chemotherapy alone for advanced gastric cancer with a single non-curable factor (REGATTA): a phase 3 , randomised controlled trial. Lancet 2016; 17: 309-18. doi: 10.1016/S14702045(15)00553-7

18. Rosenbaum PR, Rubin DB. The central role of the propensity score in observational studies for causal effects. Biometrika 1983; 70: 41-55. 10.1093/ biomet/70.1.41

19. Li M. Using the propensity score method to estimate causal effects: a review and practical guide. Organ Res Methods 2012; 15: 1-39. doi: 10.1177/1094428112447816 http://orm.sagepub.com

20. Makino $H$, Kunisaki $C$, Izumisawa $Y$, Tokuhisa $M$, Oshima $T$, Nagano $Y$, et al. Indication for hepatiec resection in treatment of liver metastasis from gastric cancer. Anticancer Res 2010; 30: 2367-76. doi: 10.1016/S14702045(15)00553-7

21. Fujitani K, Yang HK, Mizusawa J, Terashima M, Han SU, Iwasaki $Y$, et al. Gastrectomy plus chemotherapy versus chemotherapy alone for advanced gastric cancer with a single non-curable factor (REGATTA): a phase 3, randomised controlled trial. Lancet Oncol 2016; 17: 1-10. doi: 10.1016/S14702045(15)00553-7

22. Ministrini S, Bencivenga M, Solaini L, Cipollari C, Sofia S, Marino E, et al. Stage IV gastric cancer: The surgical perspective of the Italian Research Group on Gastric Cancer. Cancers 2020; 12: 1-14. doi: 10.3390/cancers12010158 